\title{
Leptospirosis following a major flood in Central Queensland, Australia
}

\author{
J. K. G. SMITH ${ }^{1 *}$, M. M. YOUNG ${ }^{2}$, K. L. WILSON ${ }^{1}$ AND S. B. CRAIG ${ }^{3,4}$ \\ ${ }^{1}$ Central Queensland Public Health Unit, Queensland Health, Rockhampton, Queensland, Australia \\ ${ }^{2}$ Wide Bay Public Health Unit, Queensland Health, Bundaberg, Queensland, Australia \\ ${ }^{3}$ WHO/FAO/OIE Collaborating Centre for Reference and Research on Leptospirosis, Queensland Health \\ Forensic and Scientific Services, Queensland Health, Brisbane, Queensland, Australia \\ ${ }^{4}$ Faculty of Science, Health and Education, University of the Sunshine Coast, Sippy Downs, Queensland, \\ Australia
}

Received 22 December 2011; Final revision 16 April 2012; Accepted 2 May 2012; first published online 25 May 2012

\section{SUMMARY}

Throughout December 2010 and January 2011, Queensland experienced widespread flooding due to unusually protracted and heavy rainfalls. In mid-January 2011, four individuals from a small community in Central Queensland were hospitalized with leptospirosis. A further five cases were subsequently identified from around Central Queensland, bringing the total to nine. Microscopic agglutination testing found that serovar Arborea (Leptospira borgpetersenii serovar Arborea) was presumptively responsible for leptospirosis in seven of nine confirmed cases. Serovars Hardjo and Australis were identified in samples from two remaining cases. All cases had exposure to flood water. No single exposure source was identified. This is the first reported outbreak of leptospirosis in Central Queensland and the first report of leptospirosis cases associated with flood water inundation in Queensland. Public health authorities should continue to promote awareness of leptospirosis in flood-affected populations. Healthcare providers must maintain a high level of suspicion for leptospirosis during and after flood events.

Key words: Community outbreaks, leptospirosis, surveillance, water-borne infections, zoonoses.

\section{INTRODUCTION}

Leptospirosis is a systemic zoonotic disease caused by spirochaetes of the genus Leptospira. Humans become infected through contact with the urine of infected animals, either directly or indirectly through contact with water or soil. In tropical and subtropical environments, the organism can survive in water and

\footnotetext{
* Author for correspondence: Dr J. K. G. Smith, Public Health Physician, Central Queensland Public Health Unit, PO Box 946, Rockhampton, Queensland 4700, Australia. (Email: James_Smith1@health.qld.gov.au)
}

soil for months [1]. Infection can occur through ingestion or via mucosal surfaces or through cuts and abrasions of the skin [2]. Globally, animal reservoir hosts include rodents, livestock and domestic pets [2] and in Australia the major reservoir hosts are rats, cattle, pigs and dogs [3]. Leptospirosis presents with a wide spectrum of clinical features. Many cases exhibit non-specific, influenza-like symptoms including fever, chills, headaches and myalgia. Complications can be severe and include hepatic and renal dysfunction, myocarditis, meningism and pulmonary haemorrhage with respiratory failure [4-6]. 
Internationally, leptospirosis tends to occur in four major settings $[1,2,7,8]$. The first is occupations that involve contact with infected animals or environments contaminated by infected animals, such as livestock, abattoir and agricultural work. The second setting is crowded urban environments where rodents are present, particularly in the presence of poverty, crowding and inadequate infrastructure including sewerage systems and drainage. The third setting, which has emerged over recent decades, is recreational exposure, including adventure tourism and watersports activities, pet ownership and gardening.

The fourth setting, also recognized in the last two decades, is natural disasters involving flooding. Flood-related outbreaks of leptospirosis have been documented in geographically diverse areas and settings [2, 6, 7, 9], and flooding is recognized as a risk factor for leptospirosis in countries including Argentina [10], Brazil [11], India [12], Lao People's Democratic Republic [13], Philippines [14], Mexico [15] and the USA [16].

In late 2010 and early 2011, the state of Queensland in Australia experienced frequent protracted and heavy rain events which led to widespread flooding. Flood levels peaked in Central Queensland between 23 December 2010 and 4 January 2011 [17]. In mid-January 2011, Central Queensland Public Health Unit (CQPHU) received a report that five individuals from a Central Queensland town with a population of around 500 people had been hospitalized with an acute febrile illness, later identified in four of those hospitalized as leptospirosis. There was significant community interest in these well-publicized cases.

The area served by CQPHU straddles the Tropic of Capricorn and contains a population of around 230000 people in an area of about $450000 \mathrm{~km}^{2}$ [18]. In this area there is usually a small number of sporadic cases of leptospirosis annually, with just 75 cases notified since 1985 .

The Central Queensland town had experienced extensive flooding over previous weeks and in January 2011 the community embarked on a large-scale cleanup operation. Further cases of febrile illness were identified in other parts of Central Queensland similarly affected by flooding in subsequent weeks. CQPHU investigated all reported potential leptospirosis cases to facilitate laboratory confirmation and to establish potential sources of exposure, including any possible common exposure.

\section{METHODS}

CQPHU established enhanced surveillance for suspected cases of leptospirosis (and other potentially flood-related infections) in flood-affected communities through regular communiqués with health service providers, in addition to usual laboratory surveillance. Community messages through various media provided public health advice regarding prevention of exposure to potentially contaminated flood water, mud and other debris.

The initial surveillance case definition for leptospirosis was: any adult or child who presented with severe febrile illness not explained by another cause, in Central Queensland, with onset of illness between 26 December 2010 and 26 March 2011. Cases were subsequently re-classified as 'confirmed', 'probable' or 'not a case' on the basis of laboratory testing.

Case investigation and collection of laboratory samples were undertaken in accordance with Queensland Health guidelines [3]. In addition to the standard notification case report form, cases were asked to complete a brief questionnaire to ascertain greater detail (than is usually collected) about residential history in the month prior to onset of illness (including temporary relocation due to flooding), consumption of food contaminated by flood water; injuries (particularly breaches to skin related to flood exposure), contact with animals; and exact details of exposure to flood water and involvement in flood recovery.

Confirmed cases required laboratory definitive evidence, in keeping with existing Queensland Health notification criteria [3]. Laboratory definitive evidence included:

- isolation of pathogenic Leptospira species, or

- a fourfold or greater rise in Leptospira microagglutination titre (MAT), or

- a single Leptospira MAT $\geqslant 400$, supported by a positive enzyme-linked immunosorbent assay IgM result.

Nucleic acid (polymerase chain reaction; PCR) testing was used early in the clinical course (before MAT could be undertaken), to identify 'probable' cases.

\section{RESULTS}

Eleven individuals met the initial surveillance case definition for suspected leptospirosis. Nine of these individuals subsequently met the criteria for a confirmed case. The nine confirmed cases observed over 
Table 1. Microagglutination titre (MAT) results for nine confirmed cases

\begin{tabular}{|c|c|c|c|c|c|}
\hline \multirow[b]{2}{*}{$\begin{array}{l}\text { Case } \\
\text { no. }\end{array}$} & \multicolumn{2}{|c|}{ Acute phase MAT } & \multicolumn{2}{|c|}{ Convalescent phase MAT } & \multirow[b]{2}{*}{$\begin{array}{l}\text { Presumptive } \\
\text { serovar }\end{array}$} \\
\hline & MAT titre & $\begin{array}{l}\text { Days from } \\
\text { illness onset }\end{array}$ & MAT titre & $\begin{array}{l}\text { Days from } \\
\text { illness onset }\end{array}$ & \\
\hline 1 & Not done & - & 800 & 12 & Arborea \\
\hline 2 & Non reactive & 4 & 400 & 13 & Arborea \\
\hline 3 & Non reactive & 5 & 3200 & 27 & Arborea \\
\hline 4 & Non reactive & 4 & 3200 & 24 & Arborea \\
\hline 5 & Non reactive & 4 & 1600 & 23 & Arborea \\
\hline 6 & Non reactive & 4 & 100 & 19 & Arborea \\
\hline 7 & Non reactive & $<1$ & 1600 & 15 & Arborea \\
\hline 8 & Non reactive & 8 & 3200 & 16 & Hardjo \\
\hline 9 & Not done & - & 800 & 24 & Australis \\
\hline
\end{tabular}

this short time period represented a substantial increase compared to the number of leptospirosis cases usually identified in Central Queensland each year. Only nine confirmed cases were observed in Central Queensland for the entire 7-year period leading up to the flood.

The median age of confirmed cases was 37 years (range 17-55 years). All nine confirmed cases were male and none of the cases were identified as Aboriginal or Torres Strait Islander. Two thirds of cases reported that their principal employment was within the agricultural sector. Two of the remaining three cases reported that they were resident on farms during the Queensland floods of 2010-2011.

Seven of the nine cases developed symptoms within a 3-day period in early January 2011, while the remaining two cases developed symptoms in the following weeks. Since all cases described multiple exposures to flood water, it was not possible to estimate incubation period in this instance. Six of the nine were able to recall the first day of exposure to flood water. Of these six cases, the median number of days between first exposure to flood water and illness onset was $20 \cdot 5$ days (range 13-31 days).

All confirmed cases were hospitalized (median length of stay 5 days, range 2-8 days) and all reported severe fever with chills and headache. Other common symptoms included nausea and myalgia. Two of the nine confirmed cases were noted to have characteristic conjunctival suffusion. All of the confirmed cases were found to have derangement of liver function tests. Two cases experienced cardiac sequelae of leptospirosis, but none experienced pulmonary haemorrhage. All confirmed cases were found to have positive PCR test results, as well as positive IgM test results and MAT results consistent with the case definition.

MAT testing found that serovar Arborea (Leptospira borgpetersenii serovar Arborea) was presumptively responsible for leptospirosis in seven of nine confirmed cases (Table 1). Serovars Hardjo and Australis were the presumptive causes of disease in the remaining two cases.

All confirmed cases reported direct exposure to flood water and flood-affected property during the Queensland flood. No single exposure source was identified. Most reported superficial injuries such as cuts and scratches on upper and lower limbs at the time of exposure to flood water. Use of personal protective equipment (PPE) was variable, with none of the confirmed cases reporting continual use of protective gloves during flood water exposure. Limited use of enclosed footwear during flood water exposure was also reported.

\section{DISCUSSION}

In the decade 2000-2009, Queensland recorded between 65 and 137 notifications of leptospirosis annually in a population that has grown from 3.6 to $4 \cdot 5$ million people over those years. Queensland notifications have accounted for $53-80 \%$ of total notifications in Australia [19]. The significant majority of Queensland notifications are from the wet tropics of northern Queensland and workers in the banana industry of the Innisfail-Tully-Cardwell area of northern Queensland account for about $25 \%$ of all reported leptospirosis cases in Queensland [20]. Other agricultural occupations account for about 
$40 \%$ of Queensland cases, and recreational activities account for a further $18 \%$ [21].

Notifications for leptospirosis are generally uncommon in Central Queensland. Unprecedented flooding in the region was temporally associated with a marked increase in the number of notifications of leptospirosis. Increased numbers of leptospirosis notifications were also seen in other flood-affected parts of Queensland and the first quarter of 2011 (1 January to 3 April 2011) saw 2.3 times the mean number of year-to-date notifications compared to the average in Queensland for the previous 4 years [22]. We defined the increase in leptospirosis cases observed in Central Queensland as an outbreak, due to the temporal association between cases and the clear geographical association between cases and flood-affected areas.

Alerts to health professionals issued during the flood highlighted the possibility of uncommon conditions such as leptospirosis and melioidosis. It is possible that an increase in awareness among both health professionals and the general public may have prompted an increase in investigation for leptospirosis; perhaps contributing to the increased number of cases detected. This effect seems most likely for cases with milder illness who may not otherwise have sought medical review and investigation. Since all nine cases in this series were hospitalized with severe febrile illnesses, it is likely that the diagnosis of leptospirosis would have been reached in most of these cases due to the more rigorous testing associated with hospital admission, irrespective of any alerts issued. We therefore believe that while increased awareness may have prompted an increase in testing in general, the observed increase in confirmed cases compared with background notification data represents a real and significant increase in leptospirosis in Central Queensland.

All nine confirmed cases from Central Queensland reported direct contact with flood water during the known incubation period for leptospirosis of 2-30 days [23]. It is presumed that infection with leptospirosis in this outbreak resulted from people wading through flood water and cleaning up after the flood in an environment contaminated by urine from animal reservoir hosts.

This is the first report of an outbreak of leptospirosis cases associated with flood water inundation in Queensland, and the first reported cluster of cases in Central Queensland. While all of the cases in this series had direct exposure to flood water and flood-affected property, no single common exposure was identified between cases.

There are 16 recognized species of Leptospira. These species can then be differentiated further into serovars, based on serological reactions to specific cellular antigens found on these motile spirochaetes. There are over 250 recognized serovars of pathogenic leptospires [5]. In this series, MAT was used to identify the infecting serovars. Since MAT cannot definitively identify an infecting serovar, the serovar detected by the MAT is often described as 'presumptive'. It should be noted that the MAT panel used in Australia has high specificity for identifying infecting serovars circulating in Australia [24]. In recent years, serovars Arborea, Zanoni and Australis have been dominant in human leptospirosis infections in Queensland [25]. Serovar Arborea was first identified in Australia in a human case of leptospirosis in 1998 [26] and is now widespread throughout Queensland and northern New South Wales [25]. It is a serovar which is generally associated with mice and rats, in particular Mus domesticus and Rattus rattus [26]. As with Arborea, serovar Australis is usually associated with rats, although it may also be associated with cattle [27]. Serovar Hardjo is generally associated with cattle [28].

In this outbreak, all of the cases were male. This is consistent with the gender distribution of leptospirosis in Australia generally. Follow-up of 883 Queensland cases over a 7-year period between 1998 and 2004 found that $93 \%$ were male [21]. The median age in the 1998-2004 follow-up study was 34 years, while in our January 2011 Queensland outbreak the median age was 37 years. Fifty-two per cent of the Queensland cases in the 1998-2004 period were hospitalized, while in our January outbreak all nine cases were admitted to hospital.

Prevention and control of leptospirosis concentrates on the 'Cover, Wash, Clean Up' approach endorsed by Queensland Health [29, 30]. This approach advocates for prevention of leptospirosis through appropriate use of PPE such as gloves and covered footwear, application of waterproof dressings for cuts and grazes, regular showering and hand hygiene and pest control measures to limit vector numbers. Simple preventive measures appear to be far more effective for prevention of leptospirosis than immunization and chemoprophylaxis.

Vaccination of individuals with specific occupational exposures has been undertaken in countries such as Italy and Spain [4], but a human vaccine for 
leptospirosis is not available in Australia. Even if a vaccine were available in Australia, it is unlikely it would have been useful in this outbreak. Vaccines available internationally induce only short-term immunity [6]. The immunity that leptospirosis human vaccines generate is specific to serovars included in the vaccines, with no cross-protective immunity $[6,23]$. The inability to identify the responsible serovar or serovars in a timely fashion, combined with other obvious limitations to the use of vaccination in situations where large proportions of the flood-affected population may be exposed to flood waters, render vaccination impractical and inappropriate for control of flood-related outbreaks such as this.

A 2009 Cochrane review by Brett-Major \& Lipnick examined the evidence around use of antibiotic prophylaxis for leptospirosis [31]. This review included three prospective, randomized trials; all of which looked at the use of doxycycline for chemoprophylaxis. Doxycyline chemoprophylaxis was associated with an increased likelihood of nausea and vomiting, but demonstrated no clear benefit in reducing the risk of leptospirosis. A WHO review article recognizes that chemoprophylaxis may be indicated for use in specific situations, but claims that there is 'no evidence to support the use of mass chemoprophylaxis during an outbreak' [6]. Given the paucity of evidence and the fact that large numbers of people would need to be (urgently) supplied with large volumes of doxycycline in a flood event, chemoprophylaxis does not appear to be a viable option for future outbreaks in Queensland.

The public health implications for future flooding events in Central Queensland are twofold. First, advice to the public should continue to emphasize the need to dress appropriately when in flood water or during flood recovery efforts. This includes wearing gloves, long-sleeved shirts and long trousers, and enclosed footwear. Injuries sustained in flood water should be thoroughly washed and dressed appropriately with waterproof dressings. This advice is in keeping with 'Cover, Wash and Clean Up' advice [29]. Public health messaging throughout the flood event emphasized these important preventive measures.

The second implication is that public health practitioners should raise awareness of leptospirosis among clinicians early during future flooding events. Clinicians in areas where leptospirosis is usually uncommon, as is the case in Central Queensland, are unlikely to be familiar with the disease. Furthermore, early leptospirosis is characterized by non-specific symptoms which may be mistaken for illnesses such as influenza or hepatitis, resulting in diagnostic delay [6]. This outbreak of an uncommon condition reinforces the need for public health practitioners to communicate regularly with hospital and primaryhealth clinicians during natural disasters in order to raise awareness of the spectrum of diseases associated with specific types of disaster. Early advice to Queensland hospitals and primary healthcare services during this flood event did mention leptospirosis, together with other illnesses known to be related to flooding such as melioidosis, food- and water-borne illnesses and vector-borne diseases.

The epidemiology of endemic leptospirosis in Australia has been well defined over the past decade. The cases described in this report serve as a potent reminder that flood waters can disseminate a variety of infectious conditions, including leptospirosis. Last, with Arborea, the most common serovar found in this outbreak, rats and mice appear to have been the predominant vectors. This lends support to provision of advice about standard rodent control measures and hygiene standards before and after contact with potentially contaminated environments.

\section{ACKNOWLEDGEMENTS}

We are very grateful to the following people for their help and assistance in investigating this outbreak: Drs Bruce Chater, Ben Rogers, Hugh Wright and Katie Goot.

\section{DECLARATION OF INTEREST}

None.

\section{REFERENCES}

1. Guerra MA. Leptospirosis. Journal of the American Veterinary Medical Association 2009; 234: 472-478.

2. Lau CL, et al. Climate change, flooding, urbanisation and leptospirosis: fuelling the fire? Transactions of the Royal Society of Tropical Medicine and Hygiene 2010; 104: 631-638.

3. Queensland Government. Communicable disease control manual: Leptospirosis. Brisbane: The State of Queensland (Queensland Health), 2010.

4. Hawker J, et al. Communicable Disease Control Handbook, 2nd edn. Oxford: Blackwell Publishing Ltd, 2005.

5. Levett PN, Haake DA. Leptospira species (leptospirosis). In: Mandell GL, Bennett JE, Donlin R, eds. 
Principles and Practice of Infectious Diseases. Philadelphia, PA: Churchill Livingstone, 2010.

6. World Health Organization. Leptospirosis : an emerging public health problem. Weekly Epidemiological Record 2011; 86: 45-52.

7. Vinetz JM. Leptospirosis. Current Opinion in Infectious Diseases 2001; 14: 527-538.

8. McBride AJ, et al. Leptospirosis. Current Opinion in Infectious Diseases 2005; 18: 376-386.

9. Trevejo RT, et al. Epidemic leptospirosis associated with pulmonary haemorrhage - Nicuragua, 1995. Journal of Infectious Diseases 1995; 178: 1457-1463.

10. Vanasco NB, et al. Clinical characteristics and risk factors of human leptospirosis in Argentina. Acta Tropica 2008; 107: 255-258.

11. Cruz LS, Vargas R, Lopes AA. Leptospirosis: a worldwide resurgent zoonosis and important cause of acute renal failure and death in developing nations. Ethnicity and Disease 2009; 19: S1-37-41.

12. Bhardwaj P, Kosambiya JK, Desai VK. A case control study to explore the risk factors for acquisition of leptospirosis in Surat city, after flood. Indian Journal of Medical Sciences 2008; 62: 431-438.

13. Kawaguchi L, et al. Seroprevalence of leptospirosis and risk factor analysis in flood-prone rural areas in Lao PDR. American Journal of Tropical Medicine and Hygiene 2008; 78: 957-961.

14. Yanagihara Y, et al. Current status of leptospirosis in Japan and Philippines. Comparative Immunology, Microbiology and Infectious Diseases 2007; 30: 399413.

15. Leal-Catellanos CB, et al. Risk factors and the prevalence of leptospirosis infection in a rural community of Chiapas, Mexico. Epidemiology and Infection 2003; 131: 1149-1156.

16. Gaynor K, et al. Leptospirosis on Oahu: an outbreak associated with flooding of a university campus. American Journal of Tropical Medicine and Hygiene 2007; 76: 882-885.

17. Australian Government. National Climate Centre, Bureau of Meteorology (http://www.bom.gov.au/climate/ current/statements/scs24b.pdf). Accessed 28 July 2011.

18. Queensland Government. Office of Economic and Statistical Research. Queensland Regional Profiles: Rockhampton region. Brisbane: The State of Queensland (Queensland Treasury), 2010.

19. Queensland Government. Leptospirosis surveillance reports (http://www.health.qld.gov.au/qhcss/lep_rep. asp). Accessed 28 July 2011.
20. Queensland Government. Queensland Health Forensic and Scientific Services. WHO/FAO/OIE Collaborating Centre for Reference \& Research on Leptospirosis. National leptospirosis surveillance report number 16 . Brisbane: The State of Queensland (Queensland Health), 2007.

21. Slack AT, et al. The epidemiology of leptospirosis and the emergence of Leptospira borgpetersenii serovar Arborea in Queensland, Australia, 1998-2004. Epidemiology and Infection 2006; 137: 1217-1225.

22. Queensland Government. Statewide weekly communicable diseases surveillance report: 4 April 2011. Brisbane: The State of Queensland (Queensland Health), 2011.

23. Heymann DL (ed). Control of Communicable Diseases Manual, 19th edn. Washington, DC: American Public Health Association, 2008.

24. Craig SB, et al. Haemoglobin and red cell counts in leptospirosis patients infected with different serovars. Revista da Sociedade Brasileira de Medicina Tropical (in press).

25. Queensland Government. Queensland Health Forensic and Scientific Services. WHO/FAO/OIE Collaborating Centre for Reference \& Research on Leptospirosis. National leptospirosis surveillance report number 18. Brisbane: The State of Queensland (Queensland Health), 2009.

26. Queensland Government. WHO/FAO/OIE Collaborating Centre for Reference \& Research on Leptospirosis (http://www.health.qld.gov.au/qhcss/qhss/lepto/ documents/arborea.pdf). Accessed 14 February 2011.

27. Queensland Government. WHO/FAO/OIE Collaborating Centre for Reference \& Research on Leptospirosis (http://www.health.qld.gov.au/qhcss/qhss/lepto/ documents/australis.pdf). Accessed 14 February 2011.

28. Queensland Government. WHO/FAO/OIE Collaborating Centre for Reference \& Research on Leptospirosis (http://www.health.qld.gov.au/qhcss/qhss/lepto/ documents/hardjo.pdf). Accessed 14 February 2011.

29. Queensland Government. Queensland Health fact sheet: Leptospirosis (http://access.health.qld.gov. $\mathrm{au} / \mathrm{hid} /$ InfectionsandParasites/BacterialInfections/ leptospirosis_fs.pdf). Accessed 14 February 2011.

30. Slack A. Leptospirosis. Australian Family Physician 2010; 39: 495-498.

31. Brett-Major DM, Lipnick RJ. Antibiotic prophylaxis for leptospirosis. Cochrane Database of Systematic Reviews 2009. Issue no. 3. Art. no. CD007342. 